\title{
Comparative Metabolome Analyses of Chinese Hickory Nut Kernels Highlight The Importance of Allocation Shift and Accumulation on Primary and Secondary Metabolites During In-Situ Germination
}

\author{
Lihong Xiao ( $\nabla$ xiaolh@zafu.edu.cn ) \\ Zhejiang A\&F University \\ Wen-Dee Ong \\ Zhejiang A\&F University \\ Jianhua Wang \\ Zhejiang A\&F University \\ Chunying Huang \\ Zhejiang A\&F University \\ Junye Hong \\ Zhejiang A\&F University
}

Jianwei Xi

Zhejiang A\&F University

Saibin Lv

Zhejiang A\&F University

Xuan Li

Zhejiang A\&F University

Jianqin Huang

Zhejiang A\&F University

Research article

Keywords: In-situ germination, seed quality, metabolomic profiling, Carya nut, Chinese hickory

Posted Date: September 17th, 2020

DOl: https://doi.org/10.21203/rs.3.rs-72375/v1

License: (c) (1) This work is licensed under a Creative Commons Attribution 4.0 International License.

Read Full License 


\section{Comparative Metabolome Analyses of Chinese Hickory Nut Kernels Highlight the Importance of Allocation Shift and Accumulation on Primary and Secondary Metabolites during In-Situ Germination}

Lihong Xiao ${ }^{1, *}$, Wen-Dee Ong ${ }^{1}$, Jianhua Wang ${ }^{1}$, Chunying Huang ${ }^{1}$, Junye Hong ${ }^{1}$, Jianwei $\mathrm{Xi}^{1}$, Saibin $\mathrm{Lv}^{1}$, Xuan $\mathrm{Li}^{1}$, and Jianqin Huang ${ }^{1}$

Affiliations: ${ }^{1}$ State Key Laboratory of Subtropical Silviculture, Zhejiang A\&F University, Hangzhou 311300, China; xiaolh@zafu.edu.cn

* Correspondence: xiaolh@zafu.edu.cn; Tel.: +86 0571-61083202; ORCID: 0000-0003-48646874 


\begin{abstract}
Background: Chinese hickory (Carya cathayensis) and pecan (C. illinoinensis) are popular nut trees with high economic value in the family Juglandaceae, due to the richness in nutrients and multiple health factors in their nut kernels. However, the considerable in-situ germination of the nut seeds severely impact the quality of the kernels. The fundamental metabolic mechanism of in-situ germination has been widely reported in major crops but rare in nut crops.
\end{abstract}

Results: We sampled non- and pre-sprouted Chinese hickory nut kernels and performed liquid chromatography-tandem mass spectrometry (LC-MS/MS)-based metabolomic analyses. Comparative metabolomic analyses revealed 356 significantly-changed metabolites during insitu germination. Detailed analysis emphasized the increasing accumulation and dynamic changes in compounds of primary mechanism and energy supply for in-situ germination. The results highlighted the importance of allocation shift and accumulation on four classes of primary metabolites and their derivatives, and two secondary metabolite classes between the transitions of germination initiation and germination and seedling establishment during the process. Metabolites related to carbohydrates and nitrogen metabolism, and few harmful to human health also demonstrated increasing accumulation along the process.

Conclusions: The findings flourish our understanding of the metabolic mechanisms of in-situ germination and will be useful for future application in molecular breeding in Chinese hickory.

Key words: In-situ germination; seed quality; metabolomic profiling; Carya nut; Chinese hickory 


\section{Background}

Seed is the main propagule for reproduction to next generation and very important to seedsbased food supply [1]. In general, yield of crops is tightly linked to seed quality, a crucial indicator for plant germination, growth and development, and future yield [2]. Dormancy breaking to start germination of orthodox seeds largely contributes to both crop yield and quality under favorable condition in the right season [3]. While in-situ germination, also defined as preharvest sprouting, i.e. precocious germination of seeds before harvest, impacts the quality and yield of crops and becomes a treat to food production [4-6]. In-situ germination led to severe annual loss of major crops worldwide. For example, occurrence of precocious germination in rice field of South China could reach up to over $6 \%$ field acreage for conventional rice and be up to $20 \%$ for hybrid rice [7]. Seed pre-germination in spikes occurs in almost every wheat growing region in the world and more than $4 \%$ on grain kernel is classified as not suitable for human consumption, which often used only as animal feedstock $[5,8]$. The sprouted wheat could be sold out at very low price and leads up to $\$ 1$ billion in annual losses3, 8. In India, in-situ germination is a big problem for pulse production, which cause about $60 \%-70 \%$ of yield losses in green gram and black gram [4]. Sprouted grains and pulse crops are of low marketable value and can be difficult to thresh from the non-sprouted grains thus leads to reduced economic return.

Our field observation on Carya nuts indicated that in-situ germination occurs not only in major crops but also nut crops. Carya (Juglandaceae) nuts especially Chinese hickory (Carya cathayensis) and pecan (C. illinoisensis) are largely consumed as they are low in saturated fatty acid and rich in unsaturated fatty acids (over $60 \%$ of the kernel dry weight), proteins, antioxidant, phenolic compounds, tocopherol and B vitamin [9]. The seeds of the hickories are enveloped with an inner brown hard endocarp and a green fleshy outer husk (exocarp and mesocarp). Early 
germination cannot be detected until the husk is removed causing a variation in the collected produce at harvest. A considerable proportion of in-situ germination were found in plantations in Zhejiang province, China for Chinese hickory and in the orchards in Texas, USA for some varieties of pecan in the harvest season of recent years (unpublished field observation; Fig. 1A, Fig. S1). Despite the highly marketable potential of Carya nut kernels, early sprouting nuts became moldy and bitter and further led to inedible. Carya nut seeds belong to recalcitrant and thus matured nuts required no dormancy for germinating. Germination of Carya nuts are highly affected by prolong storage period and their germination rate even decreased when kept in normal airy room temperature condition $\left(16.0^{\circ} \mathrm{C}\right.$ and $\left.82.4 \% \mathrm{RH}\right)$ [10]. Non-stratified Carya nuts are able to germinate and scored a rate above $85 \%$ while stored Carya nuts only can reach germination percentage comparable to non-stratified nuts after stratification or hormone treatment [10-11]. In addition, it has been reported that a temperature of between $30^{\circ} \mathrm{C}$ to $35^{\circ} \mathrm{C}$ improved germination uniformity and rate of Carya nuts11. However, seed dormancy and germination are fundamentally controlled by internal factors, thus, it is imperative to uncover the intrinsic mechanism of $i n$-situ germination to improve the nut quality. Long life cycle and hard to perform genetic and molecular operation on tree species, limited the understanding on the mechanism behind in-situ germination of the non-dormant tree nut crops, including Carya species. Recent progress in functional genomics approaches such as transcriptomics, proteomics, and metabolomics have provided us useful tools to investigate the intrinsic mechanisms of in-situ germination [12-13]. However, most of the studies on (in-situ) seed germination mechanisms focused on physiological, molecular and genetic aspects of several major crops or model plants [4-5,14-16]. In recent years, metabolomic approaches have been widely applied to explore the molecular mechanisms of seed life activities [17-21]. Metabolite quantitative trait loci (QTL) 
mapping and correlation-based metabolic network analysis connecting the morphological data revealed the central role of amino acids in coordinating interactions of other metabolites (sugars and organic acids) during tomato seed germination [17]. Another study suggested accumulation on the fatty acids (e.g. palmitate and linoleate), succinate and fumarate of thetricarboxylic acid (TCA) cycle and glycerol-3-phosphate during tomato seed germination [19]. Non-targeted metabolome study on germinating poplar seeds also revealed the crucial contribution of primary metabolite contents involving the galactose metabolism and alanine, aspartate, and glutamate metabolism [21]. Alteration on the regulations of lipids, fatty acids, amino acids, oxalate, and the raffinose family of oligosaccharides are found connected to the different germination status of dormant and susceptible wheat varieties [6]. On the other hand, participation of metabolites involving amino acids and phenylpropanoids biosynthesis are connected to the germination of rice seed under low temperature stress [22]. For antioxidant rich crop such as mangoesteen, germination involves also the accumulation of secondary metabolites such as alkaloids, flavonoids, and xanthone [20]. These reports provide us new thought for investigation of in-situ germination mechanisms of nut crops.

In this study, we applied metabolomic approach to reveal the metabolic mechanisms by characterizing the metabolomic profiles during in-situ germination of Chinese hickory embryos. Non-germinated and on tree germinated samples were used to better understand the physiological changes at metabolite level along the in-situ germination progression. The metabolic signatures identified here will offer a good phenotyping tool to plant breeders, which are also beneficial and will provide clues for future seed quality improvement in nut crops by molecular breeding. 


\section{Results}

\section{Identification and Occurrence Statistics of In-Situ Germination in Chinese Hickory Nuts}

Seed of Chinese hickory has a thin brown seed coat and enveloped by thick hard brown endocarp and an outer thick green fleshy husk [23]. In-situ germination of Chinese hickory nut occurs inside the husk and seed morphology underwent apparent changes concurrent with the germination process. As mentioned above, the collected Chinese hickory nuts were divided into four groups: non-germinated group P0 and pre-sprouted groups P1 to P3 (Fig. 1A). Morphologically, non-germinated Chinese hickory nuts (group P0) are mature seeds enveloped with hard un-cracked endocarps. Nuts with a minor crack line on endocarp or nuts with cracking endocarp but no visible radicle breaking through testa were classified as P1 group, representing the initiation of in-situ germination. As the germination proceeds, radicle would break through testa and endocarp and less than 2 mm-length white radicle was visible from the cracked endocarp of some nuts, which signify germination and classified as group P2. The next phase group P3, which were classified by those nuts having emerged radicles with over $3 \mathrm{~mm}$ in length and black radicle tips, would involve seedling establishment. To facilitate further data analyses and comparison, we assigned the transition from P0 to P1 with broken endocarp as initiation of germination (IG) phase. After the IG phase, radicle broke through the testa and protrusion, i.e. the transition from $\mathrm{P} 1$ to $\mathrm{P} 2$, representing successful seed germination, was assigned as germination $(\mathrm{G})$ phase. Nuts with dark radicle tips were deduce as samples that have completed germination and entering the seedling establishment stage. Thus, the transition from P2 to P3 stage was termed post-germination (PG) phase. The pre-sprouting of Chinese hickory nut occurs between $6.07 \%$ to $6.71 \%$ for two consecutive years (2018-2019) (Table 1), close to the preharvest sprouting ratio of conventional rice [14]. 


\section{Global Profiles of Metabolomes during In-Situ Germination}

To gain the fundamental metabolic mechanisms of Chinese hickory nut kernels during insitu germination, metabolomic profiles from samples of each germinating stage (P1 to P3) of Chinese hickory nuts were compared to non-germinated samples, P0. A total of 649 compounds were detected and 356 of them were identified as differentially-regulated metabolites in at least one of the in-situ germination phases (Fig. 1B, Table S1). Of the 356 differentially regulated metabolites, 158 significantly changed their levels in IG phase and the number increased to 290 in the $\mathrm{G}$ phase. During IG phase, 313 compounds were detected with significant level alterations. Principal component analysis (PCA) showed that samples from same group were clustered together, representing discrete pattern among the groupings (Fig. 1C). PCA plot showed that Group P0 and group P1 were separated from group P2 and P3 on the x-axes, PC1, which accounted for about $45 \%$ of the separation. When PC2 was taken into consideration, the PCA plot demonstrated that metabolites in group P1 have overlapping factors between groups, $\mathrm{P} 0$ and $\mathrm{P} 2$. The results portrayed a closer connection among groups $\mathrm{P} 0, \mathrm{P} 1$ and $\mathrm{P} 2$, while the germinating sample group P3 may be undergoing an exclusive physiological change.

Of the 158 metabolites identified during IG phase, about 90\% (142) were also involved in both $\mathrm{G}$ and PG phases (Fig. 1B). Of the 290 differentially-regulated compounds in G phase, 109 were participated in phase transitions of PG (P2 to P3). But only 10 differentially-regulated metabolites were shared by both phase transitions of IG (P0 to P1) and $\mathrm{G}$ (P1 to $\mathrm{P} 2$ ). The unique differentially-regulated metabolites of each phase transition also showed an increasing trend during the in-situ germination (IG: 4; G: 29; PG: 60), suggesting that both of radicle breaking though testa (P1 to $\mathrm{P} 2$ ) and seedling establishment (P2 to $\mathrm{P} 3$ ) needs involvement of more unique metabolites, in comparing to the initiation of germination. By comparing with non-germinated 
sample, the levels of most of the differentially-regulated compounds (296) showed an upregulated pattern and only $60(16.9 \%)$ compounds displayed a down-regulated trend in the germinated samples (P1 to P3; Fig. 1B). Among the commonly regulated compounds which showed significant level changes in all the three phases, only two compounds, the sn-glycero-3phosphocholine and the reduced form of glutathione were detected with down-regulation trend (Fig.1B; Fig. 2; Table S1). The levels of all 10 shared compounds between IG and G phases were significantly accumulated. While, increasing levels for 89 overlapping compounds were detected in the $\mathrm{G}$ and $\mathrm{PG}$ phases (Fig. 1B; Fig. 2). The results suggested that the level-up of the metabolites were necessary for the initiation of germination, radicle emergence and subsequent seedling establishment during in-situ germination.

\section{Allocation Shift on Primary and Secondary Metabolites during In-Situ Germination}

Classification of the differentially regulated metabolites showed that the alternation of compound levels induced by in-situ germination mainly involved primary metabolites, representing $62.4 \%$ $\sim 68.5 \%$ of the differentially regulated metabolites and several secondary metabolism products about $27.2 \% \sim 30.7 \%$ of the 356 significantly changed compounds (Fig.s 3A, Table S1). The analyses revealed that organic acids, lipids, amino acids and nucleotides as well as their derivatives (over $60 \%$ of the total differentially regulated metabolites) consisted the major parts of the differentially-regulated primary metabolites, and two classes of secondary metabolites, polyphenols and phenylpropanoids, were also the representatives of the most abundant categories of differentially-regulated metabolites (Fig.s 3, Table S1). Of them, apparent and opposite proportion shifts occurred on classes of these metabolites in the in-situ germination process: the decreased proportion of lipids and amino acids as well as their derivatives and the increased proportion of organic acids, nucleotides and their derivatives, polyphenols and phenylpropanoids 
from IG phase to $G$ phase. During the IG phase, about half of the differentially-regulated metabolites were classified as the lipid $(38 ; 24.1 \%)$ and amino acid $(41 ; 25.3 \%)$, while organic acid $(15 ; 9.5 \%)$, nucleotides and derivatives $(13 ; 8.2 \%)$ and other metabolite classes $(52 ; 32.9 \%)$ held the other half proportion (Fig.s 3; Table S1). When in-situ germination proceed towards G and PG stages, proportion of lipids (G: 52, 18.0\%; PG: 54, 17.3\%) and amino acids (G: 54; 18.7\%; PG: 60; 19.2\%) reduced whilst the other metabolite classes (G: 146; 50.5\%; PG: 156; $50.0 \%$ ) displayed an increment trend, except for terpenes (Fig. 3, Table S1).

\section{Accumulation on Primary Metabolites during In-Situ Germination}

As mentioned above, the lipids, amino acids and organic acids were among the highly represented metabolites class (Fig.s $2 \& 3$, Table S1). While majority of organic acids were regulated between $\mathrm{G}$ and $\mathrm{PG}$ phases, most of the differentially-regulated lipids and amino acids were regulated throughout the in-situ germination process (Fig. 3, Table S1). By integrating the levels and their sub-class, 38 (70\%) of 54 significantly-changed lipids, including saturated (8), mono-unsaturated (7) and multi-unsaturated fatty acids (30) accumulated in all stages of the insitu germination. Of them, the levels of the $\alpha$-linolenic acid, MAG (18:2), 13-HpOTrE(r), and 9Hydroxy-(10E,12Z,15Z)-octadecatrienoic acid accumulated specifically in IG and G phases but their levels did not change much from G to PG phases (Fig. 2, Table S1). The levels several saturated fatty acids significantly increased starting in $G$ phase and reached the highest levels in PG phase (Fig. 2, Table S1). Among the differentially-regulated lipids, the arachidic acid and two isoforms of LysoPC 20:1 displayed extreme accumulation by Log2FC over 10 (Fig. 2, Table S1). Moreover, a saturated fatty acid, lauric acid and an unsaturated fatty acid, cis-gondoic acid showed reduced levels particularly in the PG phase (Fig. 2). 
Majority of the differentially-regulated amino acids and derivatives exhibited increasing levels during in-situ germination, and their metabolomic profiles also highlighted the accumulation of compounds unique to each phase (Fig. 2, Table S1). The 3,4-dihydroxy-DLphenylalanine, better known as L-DOPA showed significant accumulation only in IG phase. The $\mathrm{N}$-formylmethionine (fMet), an amino acid derivative participating in the cysteine and methionine metabolism [24], was identified specifically accumulated in P2 sample (G phase). The reduced form of glutathione was the only commonly regulated amino acids with downregulation pattern across the germination process, but an oxidized form of glutathione particularly accumulated in the PG phase (Fig. 2C). Two amino acid derivatives, phenylacetyl-Lglutamine and 5-hydroxy-L-tryptophan, known to be involved in phenylalanine and tryptophan metabolism [25-26] were detected with substantial declined levels $(\log 2 \mathrm{FC}>10)$ in the PG phase (Fig. 3C; Table S1). Worthy to be mentioned, the L-(+)-Arginine, known as an essential amino acid for premature infants and liver transplantation patients [27-28] showed dramatic accumulation across the in-situ germination process (Fig. 2, Table S1).

Significant accumulation of organic acid compounds mainly occurred in G and PG phases, reflected by detection of level change on 21 organic acids between $G$ and PG phases and identification of three and seven unique compounds in G and PG phases, respectively (Fig.s 2 \& 3, Table S1). Malic acid and fumaric acid, also intermediate metabolites of TCA cycle [20], displayed significant increased level at G phase (Fig. 2, Table S1). An important component used for production of polymer and energy [29] - the 3-hydroxybutyrate, displayed reduced level particularly in the PG phase whereas the level of rosmarinic acid increased (Fig. 3). Additionally, the pantothenic acid and tartaric acid, which have been reported involving vitamins biosynthesis, recorded declined levels during post-germination. On the other hand, the level of 5-O-p- 
coumaroyl shikimic acid, an intermediate of chlorogenic acid biosynthesis [30] was significantly increased in IG and PG phase but has no obvious level change in G phase. Moreover, the kynurenic acid (KYNA), the intermediate product of tryptophan metabolic pathway that reported with function relating to memory impairment [31] was specifically accumulated in IG phase (Fig. 2, Table S1).

Of the 32 differentially-regulated nucleotides and derivatives, only nine compounds were accumulated throughout the in-situ germination process, three compounds uniquely regulated in G phase and the remaining 20 compounds were considerably accumulated during seedling establishment (G and PG phases) (Figure 2, Table S1). Of the eight differentially-regulated vitamins, six were up-regulated, involving in five B class vitamins and one vitamin C, Lascorbate (Figure 2, Table S1). Among the B class vitamins, riboflavin (VB2) and niacinamide (VB3) showed sharp increment at PG phase.

\section{Accumulation of Secondary Metabolites during In-Situ Germination}

Comparison of the metabolomic profiles of significant variable metabolites also highlighted synthesis of antioxidants, such as the polyphenols (including flavanones, flavones, flavonoids, flavanols, anthocyanins and other polyphenols) and phenylpropanoids during the in-situ germination (Figures $2 \& 3$, Table S1). These secondary metabolites especially accumulated in G and/or PG phases (Figures $2 \& 3$, Table S1). For example, the cyanidin 3-O-galactoside was not detected in P0, P1 and P2 samples but specifically accumulated in PG phase (Figure 2, Table S1). Other anthocyanins such as kuromanin also accumulated significantly in the PG phase while accumulation of petunidin 3-O-glucoside was apparent in both phases of G and PG (Figures 2, Table S1). 
Of the 31 differentially-regulated phenylpropanoids during in-situ germination of Chinese hickory nut, 12 compounds were significantly accumulated since IG phase and the rest 19 were only accumulated after IG phase (Figures $2 \& 3$, Table S1). Abundances of N-sinapoyl hydroxycoumarin and podophyllotoxin dramatically increased in the initiation stage of germination (IG phase) and kept accumulating in subsequent stages ( $\mathrm{G}$ and IG phases). One polyphenol, epiafzelechin which was not detected in P1 samples showed significant level-up in the $\mathrm{G}$ phase and being observed a higher accumulation of $\log 2 \mathrm{FC}>14$ in PG phase (Figures $2 \&$ 3, Table S1). Besides epiafzelechin, other polyphenols such as 6-gingerol and protocatechuic acid also were significantly accumulated since G phase and in PG phase the level of 6-gingerol increased while protocatechuic acid accumulate at a lower level than that detected in $G$ phase (Figures $2 \& 3$, Table S1).

Our analyses also demonstrated significant accumulation of several terpenes including the phytol, oleanolic acid, geniposidic acid, pachymic acid and maslinic acid along the in-situ germination process (Figures $2 \& 3$, Table S1). The levels of phytol and oleanolic acid were relatively high in germinated samples and oleanolic acid recorded increasing trend along IG to PG phases. Phytol level increased from IG to G phase and did not change much between IG and G phases (Figure 2, Table S1). In contrast, the level of geniposidic acid showed slight decline along the in-situ germination process (Figure 2, Table S1). The levels of pachymic acid and maslinic acid was up-regulated in initial germination period and the maslinic acid continue to accumulate to a higher level in G phase (Figure 2, Table S1).

Moreover, a sterides class compound, azadiradione displayed an extreme level spike in IG phase and the level rose slightly from IG phase to PG phase (Figure 2, Table S1), suggesting a common and yet important participation of this compound during in-situ germination of Chinese 
hickory. Two phenolamides, N1,N2,N3-p-coumaroyl-cinnamoyl-caffeoyl spermidine and spermine displayed significant increased levels at $G$ phase and their levels stayed stagnant in $G$ and PG phases (Figure 2, Table S1). But the agmatine was identified being up-regulated in G phase and accumulated at higher intensity during post-germination period. The significant levelup of $\mathrm{N}$-acetylputriscine was detected only in $\mathrm{G}$ phase and its level declined in post-germination phase. Our results suggested that polyamines may involve in common regulatory function during germination process and mostly induced between IG and PG periods. However, distinctive polyamine compound may exert different mode of function during PG stage as observed by level increased of agmatine.

\section{Involvements of Carbohydrates and Nitrogen Metabolism and Other Compounds}

Our analyses showed that 11 carbohydrates displayed varying accumulation and regulation pattern during in-situ germination of Chinese hickory (Figure 2, Table S1). Glucose, as one of the most important energy source in life activity, started to accumulate in IG phase and the level dramatically increased after germination initiation and recorded the highest level in G phase and then dropped to very low level in IG phase (Figure 4A). Fumarate and malate, intermediates of TCA cycle exhibited a similar accumulation trend to glucose (Figure 4A). The levels of other two compounds of TCA cycle - citrate and succinate, together with ribulose 5-phosphate (a compound of pentose phosphate pathway) steadily increased from IG to G phase and reached the highest levels during PG phase. The levels of threose, mannose, melezitose O-rhamnoside, galactose and N-acetyl-D-glucosamine increased in the early stage of germination, documented the highest in the G phase and declined during the PG stage (Figure 2, Table S1). Among these compounds, the level of threose displayed sharp spike between IG and G periods. Levels of panose, melezitose and maltotetraose had higher amount in non-sprout nut than sprouted nuts. In 
contrast, the relative level of glucosamine was higher in sprouted P1 and P2 samples than nonsprout sample and glucosamine level declined sharply in the PG phase (Table S1).

Moreover, several nitrogen resource-related amino acid and derivative compounds, including the main nitrogen resource in Arabidopsis [32] - glutamine and glutamate, also showed accumulation trends. Several other nitrogen resource compounds, including arginine and ornithine as well displayed increasing accumulation trend during the in-situ germination (Figure 4B). Among these, the arginine displayed highest level of accumulation irrespective of $\mathrm{G}$ phase suggesting in-situ germination of Chinese hickory nut is accompanied by mainly arginine biosynthesis. Besides, components involved in the urea cycle such as citrulline, aspartate (Figure 4B) also accumulated in sprouted nuts. The 5-Hydroxy-1,4-naphthoquinone, also named as Juglone, was detected in both sprouted and non-sprouted Chinese hickory nut kernels and the levels slightly increased in IG phase but dramatically accumulated in G and PG phases, at of $\log 2 \mathrm{FC}>19$ and $\log 2 \mathrm{FC}>22$ foldchanges respectively (Figure 2; Table S1).

\section{Discussion}

The changes of seed storage substances during seed germination and pre-sprouting have been discussed in some cereal and legume crops and model woody plant, such as rice, barley, white, mung bean and poplar [6,21,24,32]. The above reports demonstrated that active metabolism processes occurred to provide nutrients and energy reserves for seed germination, but varying metabolite-metabolite links may exist in plant species [33,34]. Metabolite profiling of maize, wheat and mung bean during sprouting projected substantial contribution of amino acids and organic acids [18,24,33]. Molecular studies on the imbibed-barley seeds have also shown that genes involved in amino acid metabolism are triggered after imbibitions36. In poplar, the involvement of amino acid metabolism is exclusive between the late stages of embryo water 
uptake to the completion of seedling growth [21]. To better understand the underlining mechanism during in-situ germination of nut crops, we explored the synergetic roles of metabolites in the nut kernels for regulation of in-situ germination of Carya species nut kernels using a comparative metabolomics approach in this study. Here, we discuss the main metabolites and their functions during the in-situ germination of Chinese hickory nut kernels.

Bewley and Black [35] suggest that hydrolysis products of main reserves in crop seeds can be mobilized rapidly and broken down enzymatically to simpler components such as glucose, amino acids, and fatty acids, which are transferred to embryos where they provide energy source and materials for seed germination. Similarly, our results also demonstrated the extensive involvement of primary metabolisms during the in-situ gemination of Chinese hickory nuts (Figures 1 - 3; Table S1). Over $60 \%$ of the changed metabolites compose of lipids, amino acids and derivatives, organic acids and derivatives, and nucleotides and indoles (Figure 3), reflecting the needs of primary metabolites in initiation and subsequent stages of in-situ germination in Chinese hickory nut seeds. Among the four class primary metabolites, lipids and amino acids and derivatives account for nearly half of the 158 differentially regulated compounds in IG phase, far greater than the proportion of organic acids and their derivatives and nucleotides and indoles $(\sim$ 18\%). In the following $\mathrm{G}$ and PG phases, the kinds of these compounds continue to increase. Interestingly, a significant allocation shift between the classes of lipids and amino acids as well as their derivatives $(\sim 36 \%)$ and the classes of organic acids and derivatives and nucleotides and indoles $(22 \%-24 \%)$ has been detected, with a combination ratio shift of the lipids plus amino acids and derivatives: organic acids and derivatives plus nucleotides and indoles from a 2.8:1 ratio in $\mathrm{IG}$ phase to a $1: 1.5$ ratio in $\mathrm{G}$ or to a $1: 1.6$ ratio in $\mathrm{PG}$ phase. This findings suggest that more materials and energy conditions in the initiation stage need to be prepared for the later 
germination processes. Additionally, the shift is also accompanied by increased proportion and kinds on secondary metabolites, especially polyphenols and phenylpropanoids (Figure 3A), might reflecting the needs of increasing adaptability to complex environments after germination for the new potential seedling. These results also suggest that the alteration on levels of primary metabolites during initial germination stage might contribute to and trigger the synthesis of secondary metabolites at later germination to new seedling establishment stages. And our analyses demonstrate the co-ordination and interplay among primary and secondary metabolites to be imperative during the in-situ germination process of Chinese hickory nut seeds.

Of the differentially regulated primary metabolites, two compounds, L-DOPA and kynurenic acid were observed to accumulate specifically in IG phase and therefore could be used as biomarkers related to early germination in Chinese hickory (Figure 2, Table S1). L-DOPA as an allelochemical largely exudes by legumes to environment, which produces in leaves and seeds of velvet bean and releases by legumes interferes with growth of neighboring plants by causing injury to root growth [36-37]. In maize, L-DOPA inhibits the activities of phenylalanine ammonia-lyase and tyrosine ammonia-lyase causing accumulation of phenylalanine and tyrosine, which contribute to enhance lignin deposition of root cell wall and a reduction of root growth [38]. Moreover, L-DOPA as a non-protein amino acid is a precursor of many alkaloids and involves in plant secondary metabolite synthesis [36].

Despite available reports on L-DOPA accumulation in dark-germinated Phaseolus vulgaris seeds [39], there are not much knowledge of L-DOPA roles on in-situ germination. Integrating the previous reports and our results, we deduce that the considerable accumulation of L-DOPA in the early stage of in-situ germination may be important for the synthesis of secondary metabolites in the following germination phase. Meanwhile, the accumulation of L-DOPA in IG 
phase might serve as the initiator of the in-situ germination in Chinese hickory nuts, and the break of endocarp in the IG phase and subsequently radicle protrusion from seed coat in G phase would be in tight association with weakening of the cell wall and testa broken which contributed by elevated L-DOPA.

Kynurenic acid (KYNA) as a natural organic acid is first found in urine and widely existed in various types of food including plant species and which can also be generated in the brain along the kynurenine pathway in human and animals as an antagonist of ionotropic glutamate receptors and $\alpha 7$-nocotinic receptors [40]. Studies on function of KYNA in human and animals suggested its positive properties such as anti-ulcerative, antioxidative and anti-inflammatory [40]. KYNA act as an intermediate product of L-tryptophan degradation and an antagonist at excitatory glutamate receptors inhibiting the over-excitation of glutamatergic circulation [41-42]. We postulate that the accumulation of KYNA particularly at beginning of in-situ germination in Chinese hickory nuts is likely related to the spike of nitrogen content composing of arginine, glutamine, glutamate and ornithine in germinated samples. However, a recent report suggested that KYNA negatively modulates age-induced learning impairment [31]. Therefore, from the view of human health, the pre-germinated Chinese hickory kernels would not be edible.

Additionally, fMet is an amino acid coded by AUG codon, the start codon for protein synthesis [43]. The detection of up-regulation of fMet in G phase and keep at a high level in PG phase implied changes in the cellular and physiological owing to commence of protein synthesis which take place basically during the seedling establishment. Our analyses demonstrate that insitu germination of Chinese hickory mainly trigger increasing synthesis of saturated fatty acids despite detection of $\alpha$-linolenic acid with accumulating amount during initial germination and germination stages and the considerable regulation on some kinds of unsaturated fatty acids 
converted to saturated ones (Figure 3; Table S1), which may suggest their contribution as energy resource in the in-situ germination process.

Our metabolomic analyses also support involvement of glutathione, phenylalanine, tryptophan, and carbon and nitrogen metabolism during in-situ germination of Chinese hickory nut seed. The presence of glutathione in plants is important for oxidation-reduction reactions [44]. In-situ germination of Chinese hickory nut is accompanied by regulation of glutathione homeostasis. The varying regulation and level of the different forms of gluthatione suggests their critical role in protection of the -SH group proteins against oxidation during in-situ germination of Chinese hickory nut. Increased levels of oxidized glutathione in the PG phase probably provide protection for new established seedlings and support the increasing synthesis of aromatic compounds (e.g. rosmanic acid) after radicle protrusion from endocarp of Chinese hickory nut. Rosmarinic acid presumably accumulated in plant as defense compounds and has biological properties including antioxidant, antibacterial, antiviral and anti-inflammatory activities [4548].Their accumulation may exert varying physiological effects such as mitotic division, chromosomal and possible induction of DNA damage at high dose level [49]. The level-up of rosmanic acid and oxidized glutathione may explained the exclusive physiological change of P3 samples (Figure 2B) that are likely connected to the increasing abundance of reactive oxygen species (ROS)-related compounds in the post-germination stage.

ROS is linked to loss of dormancy [50] and is known responsible of oxidative damage, delays of seed germination, loss of viability during seed ageing and ROS can control plant development [49-52]. Therefore, accumulation of ROS-related compounds may suggest its roles as a secondary messenger to activate stress-responsive genes for possible seedling growth in subsequent stage. Phenylalanine has an essential interconnector role between primary and 
secondary metabolism [53]. Protrusion of radicle from seed coat is accompanied by abundant accumulation of dipeptide phenylalanine which might be essential for channeling of carbon sources to biosynthesis of phenylpropanoids. During post-germination period however, physiological change involving tryptophan metabolisms might take place as observed by an opposite regulation of 5-hydroxy-L-tryptophan, precursor for melatonin biosynthesis. Melatonin in plants participates in regulating seed germination, plant growth and development and coping with various environmental stresses [54].

It has been reported that the central of primary metabolism involves carbohydrate and nitrogen metabolism incorporating respiratory pathways to regulation seed germination [20, 55]. The balance of carbon and nitrogen in seeds affects seed development, maturation and germination statuses [6,56-57]. During seed germination, the disturbance of endogenous $\gamma$ aminobutyric acid or activation of $\alpha$-amylase activities contributes to starch hydrolysis [7,33,5758]. Carbohydrate degradation which accompanied by the accumulation of monosaccharides is expected to support germination [33]. Germinating seeds of tomato also accumulate a higher level of sucrose but a declined in glucose level [59], and the sprouting process of mung bean is accompanied by increasing level of glucose and fructose [24]. Whereas our analysis demonstrates increasing degradation of carbohydrates such as panose, melezeitose and maltotetraose during in-situ germination of Chinese hickory nut, coupled with increased level of glucose and galactose, predominantly in the G phase. Besides carbohydrates, TCA activity which converts organic matters in plants has close association with energy generation during seed germination [52]. TCA cycle plays a central role in generating ATP and Intermediates of TCA have been reported to accumulate during seed priming germination $[55,60]$. The accumulation patterns of several compound involved in TCA cycle suggest that activation of many energy 
requiring metabolic reactions occurs in $\mathrm{G}$ phase of in-situ germination. These energy sources are most likely essential to support germination process and the build-up of sugar molecules might be needed to support radicle protrusion during germination process of Chinese hickory.

Nitrate can enhance ABA catabolism and inhibit ABA synthesis [61]. Additionally, there are significant evident that the gibberellin (GA) biosynthesis has close relation to nitrate level in seed germination [57,62]. Elevated of nitrogen sources including arginine, glutamate, glutamine and ornithine (Figure 4) may have significant contribution to early germination in Chinese hickory nuts. Nitrogen in plant exists in different forms. For example, Arabidopsis uses glutamine and glutamate as the main nitrogen source, and arginine stored in seed is degraded into transportable forms such as glutamine or glutamate [62]. Our metabolomic profiles demonstrate early germination of Chinese hickory is accompanied by abundance synthesis of arginine. The role of GA in seed germination promotion is well-documented [63]. The tremendous build-up of arginine $(\log 2 \mathrm{FC}>15)$ upon break of endocarp may be related to high nitrate content promotion of ABA catabolism and activation regulation of GA biosynthesis, thus inducing seed germination. These results suggest a lower carbon to nitrogen fraction in seeds of Chinese hickory may consequent to pre-sprouting event.

Collectively, our metabolome analyses suggest that the metabolic regulation of in-situ germination in Chinese hickory nuts are similar to, but not equal to that in cereal and several other crop seeds in some extent. The in-situ germination of Chinese hickory nuts involve alteration and level changes of primary metabolites and later accumulating of secondary metabolites. The transition from non-germinate to crack of endocarp and then emergence of radicle from testa are engaged with shift on allocation of lipids, amino acids and derivatives, organic acids and derivatives, and nucleotides and indoles. Crack of the endocarp layer may be 
associated with the decrease of cell wall integrity by the build-up of L-DOPA and high nitrate level induced ABA catabolism and GA biosynthesis. Subsequently, emergence of radicle breaking through testa are associated with increase of carbon flux activity involving TCA cycle, activation of protein synthesis and secondary metabolites biosynthesis such as the phenylalanines and antioxidant polyphenols. The elevated levels of aromatic compounds, rosmanic acid and cyanidin 3-O-galactoside, both having antioxidant properties suggest increasing ROS and activation of redox signaling may be required to support further development of germinated seeds. The current study depicted the metabolic signatures in stages of in-situ germination progression of Chinese hickory nuts and provides additional evidence that the impossibility to link a phenotype to a single metabolite. Indeed, combinations and strong correlation of a set of metabolites with distinct functional characteristic can better exemplify the complexity of seed germination. Moreover, although the in-situ germination makes the difficulty for storage and the increased levels of several compounds harmful to human health and leads to inedible Chinese nuts kernels, it is also worthy to consider that the significant increase on the content of the amino acids and derivatives as well as antioxidant secondary compounds from a view of nutritional aspect. Therefore, our findings here will shed a light to the future molecular breeding to obtain the improved Carya varieties with less harmful components but more abundant nutritional components, such as the essential amino acid - arginine and the anti-tumor component - juglone, whatever with or without in-situ germination occurred in the nuts.

\section{Conclusions}

In the current study, a comprehensive metabolomes of in-situ germination in Chinese hickory nut kernels were presented. Comparative analysis identified a number of differentially regulated metabolites during the in-situ germination process. Our data can act as important basic resource 
for the functional validation of metabolites and a starting point for investigations into the molecular basis of in-situ germination in the future molecular breeding of Carya nut quality.

\section{Materials and methods}

Plant Materials. To investigate the occurrence rate and the metabolism mechanism underlying in-situ germination in Chinese hickory nuts, 20 eight- to ten-year-old trees were randomly selected from a plantation (Latitude: 30¹2’35.0450928’; Longitude: 119³8'47.385366”; Altitude: 101m) in Lin'an district, Zhejiang Province, China. At least 20 well-developed fruits were collected from each tree on early September, 2018 and 2019. First, the husk of collected samples was removed and all endocarps were screened visually. To facilitate analyses and comparison, we assigned the germinated nuts into three groups: those with crack lines on the endocarps were grouped under P1, those with cracked endocarps and visible white radicle (the length was less than the thick of endocarps, not exceed $2 \mathrm{~mm}$ ) were grouped under P2 and those with cracked endocarps and 3-5 mm-length dark radicle were grouped under P3 (PG: postgermination) (Fig. 1A). Nuts with no crack lines were used as control group (P0).

The germinated nuts collected in 2019 and 2019 were recorded, and the annual in-situ germination rate was calculated by dividing sum of P1 to P3 by the total amount of P0 to P3. For each group, three nut kernels (mainly cotyledons) from different trees, acting as three biological replicates were snap frozen with liquid nitrogen and stored at $-80^{\circ} \mathrm{C}$ until for metabolites extraction.

Metabolite Extraction and Profiling. Frozen samples were ground into fine powder and $0.1 \mathrm{~g}$ of grounded sample was mixed with $1 \mathrm{~mL}$ methanol (Merck, Germany) and incubated overnight at refrigerate condition to extract total metabolites. Mixture was then centrifuged at $10,000 \mathrm{~g}$ for 10 min and clear upper phase supernatant was recovered. Supernatant acquired was vacuum- 
dried to eliminate extraction solvent and final extracts was filtered with $0.22 \mu \mathrm{m}$ membrane before subjected to HPLC analysis. Metabolites were separated using Ultra Performance Liquid Chromatography system (Shimpack UFLC SHIMADZU CBM30A, Japan) equipped with a Waters ACQUITY UPLC HSS T3 C18 column. For liquid chromatography, parameters were set as: injection volume of $2 \mu \mathrm{L}$, column temperature at $40^{\circ} \mathrm{C}$ and flow rate of $0.4 \mathrm{~mL}$ min-1. Separated compounds were detected using tandem mass spectrometry system (Applied Biosystems 6500 QTRAP) with an electrospray ionization (ESI) source. The drying gas (nitrogen) was set at $25 \mathrm{psi}$ and with gas temperature of $500^{\circ} \mathrm{C}$. The voltage of ESI spray was fixed at $4.4 \mathrm{kV}$.

Metabolites Identification and Data Analysis. The mass spectrometry peaks were identified using Analyst 1.6.3 software (Sciex). The identification of entities was based on comparisons to in-house and public metabolite databases including ChEBI [64]and MetaboLights [654]. Prior to detail analysis, isotopic and broken signals were removed from data. The principal component analysis (PCA) and hierarchical clustering analysis were performed to assess overall data quality. For differential metabolite analyses, both univariate and multivariate analyses were applied. The univariate, Analysis of variance (one-way ANOVA) corrected with Duncan'test $(\mathrm{p}<0.05)$ was employed to monitor pattern of accumulation for each metabolites. To better characterize the relations between metabolites and their orchestrated behaviour in relation to germination process, multivariate analysis was applied. Data was subjected to partial least squares to latent structurediscriminant analysis (PLS-DA) and orthogonal partial least squares to latent structurediscriminant analysis (OPLS-DA) using SIMCA-P 14.1 (Umetrics, Sweden). Metabolites scoring either $\log 2$ fold change $\geq 1$ or fold change $\leq-1$ and with VIP (variable importance in project) value $\geq 1$ were selected as significantly-changed metabolites. The relative levels visualization of 
significantly changed metabolites were generated by the Multiple Experiment Viewer (MeV, V.4.9.0, http://mev-tm4.org/). Selected metabolites were portrayed for metabolomic interactions using MetScape 3.1 (metscape.ncibi.org/). The KEGG (Kyoto Encyclopedia of Genes and Genomes) database (URL) (http://www.genome.jp/kegg/) was also used for ID referencing and metabolic pathway searches to further analyze their biological functions.

\title{
Supplementary information
}

Additional file 1: Figure S1. Pre-sprouted pecans observed in orchards of Pecan Breeding and Genetics, Agricultural Research Service, United States Department of Agriculture, Texas, USA.

Additional file 2: Table S1.The list of identified metabolites in each in-situ germination phase of Chinese hickory.

\begin{abstract}
Abbreviations
ABA, abscisic acid; fMet, N-formylmethionine; G, germination; GA, gibberellin; IG, Initial germination; KYNA, kynurenic acid; L-DOPA, 3,4-dihydroxy-DL-phenylalanine; OPLS-DA, orthogonal partial least squares to latent structure-discriminant analysis; PCA, principal component analysis; PG, post-germination; QTL, quantitative trait loci; ROS, reactive oxygen species; TCA, tricarboxylic acid.
\end{abstract}

\section{Acknowledgments}

We would like to thank Dr. Xinwang Wang at Pecan Breeding and Genetics, Agricultural Research Service, United States Department of Agriculture for his help of providing us the photos of in-situ germinated pecan nuts. 


\section{Authors' contributions}

LX received the project and designed the experiments. LX, WDO, JW, CH, JH, JX, SL and XL, conducted the field survey and collected the samples. LX and WDO analyzed the data and wrote the draft. LX reviewed and edited the final manuscript.

\section{Funding}

This research was mainly funded by the National Science Foundation of Zhejiang Province (grant no. Z20C160001), and partially funded by the Chinese Ministry of Science and Technology (grant no. 2018YFD1000604) and Zhejiang A\&F University (grant no. 2018FR002.

\section{Ethics approval and consent to participate}

This project uses plant materials and does not utilize transgenic technology. The authority responsible for the Carya cathayensis resources is the Mount Tianmu National Nature Reserve, who provided permission to collect the samples for our scientific research.

\section{Competing of interest}

The authors declare no conflict of interest.

\section{References}

1. Larson G, Piperno DR, Allaby RG, Purugganan MD, Andersson L, Arroyo-Kalin M, et al. Current perspectives and the future of domestication studies. PNAS 2014, 111: 6139-6146.

2. Domergue J-B, Abadie C, Limami A, Way D, Tcherkez G. Seed quality and carbon primary metabolism. Plant Cell Environ 2019, 42: 2776-2788.

3. Nonogaki H, Barrero JM, Li C. Editorial: Seed dormancy, germination and pre-harvest sprouting. Front Plant Sci 2018, 9:1783-1786. 
4. Singh P, Chourasiya VK, Bajpai R, Yadav RDS. Induction of dormancy in mungbean - A review. Int J Pure App Biosci 2017, 5:639-648.

5. Simsek S, Ohm J.B, Lu H, Rugg M, Berzonsky W, Alamri MS, et al. Effect of pre-harvest sprouting on physicochemical properties of starch in wheat. Foods 2014, 3:194-207.

6. Das A, Kim D-W, Khadka P, Rakwal R, Rohila JS. Unraveling key metabolomic alterations in wheat embryos derived from freshly harvested and water-imbibed seeds of two wheat cultivars with contrasting dormancy status. Front Plant Sci 2017, 8:1203-1219.

7. Du C, Chen W, Wu Y, Wang G, Zhao J, Sun J, et al. Effects of GABA and vigabatrin on the germination of Chinese Chestnut recalcitrant seeds and its implications for seed dormancy and storage. Plants 2020, 9:449-464.

8. Black M, Bewley JD, Halmer, P. The encyclopedia of seeds: science, technology and uses. CABI. 2006.

9. Huang Y, Xiao L, Zhang Z, Zhang R, Wang Z, Huang C, et al. The genomes of pecan and Chinese hickory provide insights into Carya evolution and nut nutrition. GigaScience 2019:8, 1-17.

10. Dalkilic Z. Effects of drying on germination rate of pecan seeds. J Food Agric Environ 2013, 11:879-882.

11. Li S, Yang J, Fan Z Xi, X Chen H. Treatments for germination of Carya illinoinensis seeds. Journal of Zhejiang A\&F University 2011, 28:444-449.

12. Gao F, Ayele BT. Functional genomics of seed dormancy in wheat: advances and prospects. Front Plant Sc. 2014, 5:458. 
13. Dong K, Zhen S, Cheng Z, Cao H, Ge P, Yan Y. Proteomic analysis reveals key proteins and phosphoproteins upon seed germination of wheat (Triticum aestivum L.). Front Plant Sci 2015, 6: 107.

14. Guo L, Zhu L, Xu Y, Zeng D, Wu P, Qian Q. QTL analysis of seed dormancy in rice. Euphytica 2004. 140:155-162.

15. Zhang C, Zhou L, Lu Y, Yang Y Feng L, Hao W, et al. Changes in the physiochemical properties and strarch structures of rice grains upon pre-harvest sprouting. Carbohydrate Ploymers 2020, 234:115893.

16. Vetch JM, Stougaard RN, Martin JM, Giroux MJ. Review: Revealing the genetic mechanisms of pre-harvest sprouting in hexaploidy wheat (Triticum aestivum L.). Plant Science 2019, 281:180-185.

17. Toubiana D, Semel Y, Tohge T, Beleggia R, Cattivelli L, Rosental L, et al. Metabolic profiling of a mapping population exposes new insights in the regulation of seed metabolism and seed, fruit, and plant relations. PLoS Genet 2012, 8:e1002612.

18. Feenstra AD, Alexander LE, Song Z, Korte AR, Yandeau-Nelson, M.D, Nikolau, B.J, Lee, Y.J. Spatial mapping and profiling of metabolite distributions during germination. Plant Physiol 2017, 174:2532-2548.

19. Kazmi RH, Willems LAJ, Joosen RVL, Khan N, Ligterink W, Hilhorst HWM. Metabolomic analysis of tomato seed germination. Metabolomics 2017, 13:145-162.

20. Mazlan O, Aizat WM, Aziz Zuddin NS, Baharum SN, Noor NM. Metabolite profiling of mangosteen seed germination highlights metabolic changes related to carbon utilization and seed protection. Scientia Horticulturae 2019, 243:226-234. 
21. Qu C, Chen J, Cao L, Teng X, Li J, Yang C, et al. Non-targeted metabolomics reveals patterns of metabolic changes during poplar seed germination. Forests 2019, 10:659-171.

22. Yang M, Yang J, Su L, Sun K, Li D, Liu Y, et al. Metabolic profile analysis and identification of key metabolites during rice seed germination under low-temperature stress. Plant Sci 2019, 289:110282-110295.

23. Zhang Q, Hua HK, Huang YJ, Han KY, Xv HW, Shen YQ, et al. The relationship between developmental stages of zygotic embryos at explanting and embryogenic frequency on hickory (Carya cathayensis Sarg.). Scientia Horticulturae 2012, 139:66-70.

24. Jom K, Frank, T, Engel K-H. A metabolite profiling approach to follow the sprouting process of mung beans (Vigna radiata). Metabolomics 2011, 7:102-117.

25. Hoshino T, Yamamoto M., Uchiyama T. Formations of (5-Hydroxy)indole S-(-)-lactic acid, N-Acetyl-5-hydroxy-L-tryptophan, and (5-Hydroxy)indole carboxylic Acid in the metabolism of tryptophan and 5-hydroxytryptophan by Chromobacterium violaceum. Bioscience, Biotechnology, and Biochemistry 2014. 57:1609-1610.

26. Kaufman S. A model of human phenylalanine metabolism in normal subjects and in phenylketonuric patients. PNAS 1999. 96:3160-3164.

27. Amin HJ, Zamora SA, McMillan DD, Fick GH, Butzner DB, Parsons HG, et al. Arginine supplementation prevents necrotizing enterocolitis in the premature infant. The Journal of pediatrics 2002. 40:425-431.

28. Rabier D, Narcy C, Bardet J, Parvy P, Saudubray JM, Kamoun P. Arginine remains an essential amino acid after liver transplantation in urea cycle enzyme deficiencies. Journal of inherited metabolic disease 1991. 277-280. 
29. Bohmert K, Balbo I, Steinbüchel A, Tischendorf G, Willmitzer L. Constitutive expression of the $\beta$-ketothiolase gene in transgenic plants. A major obstacle for obtaining polyhydroxybutyrate-producing plants. Plant Physiol 2002. 128:1282-1290.

30. Kühnl T, Koch U Heller W, Wellmann E. Chlorogenic acid biosynthesis: Characterization of a light-induced microsomal 5-O-(4-coumaroyl)-d-quinate/shikimate 3'-hydroxylase from carrot (Daucus carota L.) cell suspension cultures. Archives of Biochemistry and Biophysics 1987. 258:226-232.

31. Vohra M, Lemieux GX, Lin L, Ashrafi K. Kynurenic acid accumulation under lies learning and memory impairment associated with aging. Genes and Development 2018. 32:14-19.

32. Kang J, Turano FJ. The putative glutamate receptor 1.1 (AtGLR1.1) functions as a regulator of carbon and nitrogen metabolism in Arabidopsis thaliana. PNAS 2003. 100:6872-6877.

33. Han C, Zhen S, Zhu G, Bian Y, Yan Y. Comparative metabolome analysis of wheat embryo and endosperm reveals the dynamic changes of metabolites during seed germination. Plant Physiol Biochem 2017, 115:320-327.

34. Sreenivasulu N, Usadel B, Winter A, Radchuk V, Scholz U, Stein N, et al. Barley grain maturation and germination: metabolic pathway and regulatory network commonalities and differences highlighted by new MapMan/PageMan profiling tools. Plant Physiol 2008, $146: 1738-1758$

35. Bewley J, Black M. Seeds: Physiology of development and germination. New York and London USA: Plenum Press 1994, 67-73.

36. Soares AR, Marchiosi R, Siqueira-Soares RC, de Lima RB, dos Santos WD, Ferrarese-Filho O. The role of L-DOPA in plants. Plant Signal. Behav 2014, 9:e28275. 
37. Nishihara E, Parvez MM, Araya H, Kawashima S, Fujii Y. L-3-(3,4dihydroxyphenyl)alanine (L-DOPA), an allelochemical exuded from velvetbean (Mucuna pruriens) roots. Plant Growth Regul 2005, 45:113-120.

38. Siqueira-Soares RC, Soares AR, Parizotto AV, Ferrarese MLL, Ferrarese-Filho O. Root growth and enzymes related to the lignification of maize seedlings exposed to the allelochemical L-DOPA. The Scientific World Journal 2013, 134237-134243.

39. Rahmani-Nezhad S, Dianat S, Saeedi M, Tehrani MB, Ghadiri A, Hadjiakhoondi A. Evaluating the accumulation trend of L-dopa in dark-germinated seeds and suspension cultures of Phaseolus vulgaris L. by an efficient UV-spectrophotometric method. Quim. Nova 2018, 41:386-393.

40. Turski MP, Turska M, Kocki T, Turski WA, Paluszkiewicz P. Kynurenic acid content in selected culinary herbs and spices. J Chem 2015, 617571-617577.

41. Sas K, Szabó E, Vécsei L. Mitochondria, oxidative stress and the kynurenine system, with a focus on ageing and neuroprotection. Molecules 2018, 23:191-229.

42. Juhász A, Caspó E, Ungor D, Tóth GK, Vécsei L, Dékány I. Kinetic and thermodynamic evaluation of kynurenic acid binding to GluR1270-300 polypetide by surface Plasmon resonance experiments. J Phys Chem B 2016,120:7844-7850.

43. Thach RE, Dewey KF, Brown JC, Doty P. Formylmethionine codon AUG as an initiator of polypeptide synthesis. Science 1966, 153:416-418.

44. Cnubben NHP, Rietjens IMCM, Wortelboer H, Zanden J, Bladeren PJ. The interplay of glutathione-related processes in antioxidant defense.Environ Toxicol Pharmacol 2001, 10:141-152.

45. Petersen M, Simmonds MSJ. Rosmarinic acid. Phytochemistry 2003, 62:121-125. 
46. Boonyarikpunchai W, Sukrong S, Towiwat P. Antinociceptive and anti-inflammatory effects of rosmarinic acid isolated from Thunbergia laurifolia Lindl. Pharm Biochem Beh 2014, 124:67-73.

47. Petersen M, Abdullah Y, Benner J, Eberle D, Gehlen K, Hücherig S, et al. Evolution of rosmarinic acid biosynthesis. Phytochemistry 2009, 70:1663-1679.

48. Liman R, Ciğerci IH, Gökce S. Cytogenetic and genotoxic effects of rosmaniric acid on Allium cepa L. root meristem cells. Food Chem Toxicol 2018, 121:444-449.

49. Foreman J, Demidchik V, Bothwell JHF, Mylona P, Miedema H, Torres MA, et al. Reactive oxygen species produced by NADPH oxidase regulate plant cell growth. Nature 2003, 422:442-446.

50. Oracz K, El Maarouf-Bouteau H, Kranner I, Bogatek R, Corbineau F, Bailly C. The mechanisms involved in seed dormancy alleviation by hydrogen cyanide unravel the role of reactive oxygen species as key factors of cellular signalling during germination. Plant Physiol 2009, 150:494-505.

51. El-Maarouf-Bouteau H, Mazuy C, Corbineau F, Bailly C. DNA alteration and programmed cell death during ageing of sunflower seed. J Exp Bot 2011, 62:5003-5011.

52. Bailly $\mathrm{C}$. The signalling role of ROS in the regulation of seed germination and dormancy. Biochem J 2019, 476:3019-3032.

53. Pascual MB, El-Azaz J, de la Torre FN, Canas RA, Avila C, Cánovas FM. Biosynthesis and metabolic fate of phenylalanine in conifers. Front Plant Sci 2016, 7:1030-1043.

54. Hardeland R. Melatonin in plants - diversity of levels and multiplicity of functions. Front Plant Sci 2016, 7:198-212. 
55. Wang L, Cui D, Zhao X, He M. The important role of the citric acid cycle in plants. Gen Appl Biol 2017, 8:25-29.

56. Wang L, Fu J, Li M, Fragner L, Weckwerth W, Yang P. Metabolomic and proteomic profiles reveal the dynamics of primary metabolism during seed development of lotus (Nelumbo nucifera). Front Plant Sci 2016, 7:750-762.

57. Osuna D, Prieto P, Aguilar M. Control of seed germination and plant development by carbon and nitrogen availability. Front Plant Sci 2015, 6:1023-1037.

58. Sun J, Jia H, Wang P, Zhou T, Wu Y, Liu Z. Exogenous gibberellin weakens lipid breakdown by increasing soluble sugars levels in early germination of zanthoxylum seeds. Plant Sci 2019, 280:155-163.

59. Rosental L, Perelman A, Nevo N, Toubiana D, Samani T, Batushansky A, et al. Environmental and genetic effects on tomato seed metabolic balance and its association with germination vigor. BMC Genomics 2016, 17:1047-1068.

60. Angelovici R, Fait A, Fernie AR, Galili G. A seed high-lysine trait is negatively associated with the TCA cycle and slows down Arabidopsis seed germination. New Phytol 2011, 189:148-159.

61. Ali-Rachedi S, Bouinot D, Wagner M-H, Bonnet M, Sotta B, Grappin P, et al. Changes in endogenous abscisic acid levels during dormancy release and maintenance of mature seeds: studies with the Cape Verde Islands ecotype, the dormant model of Arabidopsis thaliana. Planta 2004, 219:479-488.

62. Matakiadis T, Alboresi A, Jikumaru Y, Tatematsu K, Pichon O, Renou J-P, et al. The Arabidopsis abscisic acid catabolic gene CYP707A2 plays a key role in nitrate control of seed dormancy. Plant Physiol 2009, 149:949-960. 
63. Gubler F, Millar AA, Jacobsen JV. Dormancy release, ABA and pre-harvest sprouting. Curr. Opin.Plant Biol 2005. 8:183-187.

64. Hastings J, Owen G, Dekker A, Ennis M, Kale N, Muthukrishnan V, et al. ChEBI in 2016: Improved services and an expanding collection of metabolites. Nucleic Acids Res 2016, 44:D1214-D1219.

65. Kale NS, Haug K, Conesa P, Jayseelan K, Moreno P, Rocca-Serra P, et al. Metabolights: An open-access database repository for metabolomics data. Curr. Protoc. Bioinform 2016, 53:118.

\section{$1 \quad$ Figure legends}

2 Figure 1. Morphology and overview metabolomic data of Chinese hickory nut during in-situ

3 germination. (A) Phenotype and sample grouping of pre-sprout hickory nuts. Non-germinated 4 nut were assigned as P0. Pre-sprouted nuts with cracked lines on shell were classified as P1, 5 those with white radicle emerged from seed coat were grouped as P2 and pre-sprouts with black 6 radicle tip were grouped as P3. (B) Venn diagram of differentially regulated metabolites in each 7 in-situ germination stages. (C) Principal component analysis (PCA) plot showing the distribution 8 of non-germinated (P0) and germinated Chinese hickory nut samples (P1-P3). 
9 Figure 2. Relative levels and dynamics of differentially regulated metabolites identified during 10 in-situ germination process. The bar indicates the relative levels by $\log 2[\mathrm{FC}]$.

11 Figure 3. Classification of differentially-regulated and their distribution in each in-situ 12 germination phases. (A) The Overview of the differentially-regulated classes and their 13 proportions in IG, G and PG phases. (B) Distribution of differentially regulated lipids, amino 14 acids and derivatives, organic acid and derivatives, nucleotides and derivatives, polyphenols and 15 phenylpropanoids during the in-situ germination process.

16 Figure 4. Dynamic patterns of metabolites involved in managing carbon resource and nitrogen 17 during $i n$-situ germination. (A) The relative abundance of metabolites involved in the glycolysis, 18 pentose phosphate pathway and TCA cycle. (B) The relative abundance of major nitrogen 19 compounds contributors and those involved in urea cycle. 


\section{Tables}

Table 1: Statistics of in-situ germination occurrence in Chinese hickory.

\begin{tabular}{|c|c|c|c|c|c|}
\hline \multicolumn{3}{|l|}{ Year 2018} & \multicolumn{3}{|l|}{ Year 2019} \\
\hline $\begin{array}{l}\text { Non-germinated } \\
\text { nuts (P0) }\end{array}$ & $\begin{array}{l}\text { Germinated } \\
\text { nuts }(\mathrm{P} 1+\mathrm{P} 2+\mathrm{P} 3)\end{array}$ & Total nuts & $\begin{array}{l}\text { Non-germinated } \\
\text { nuts (P0) }\end{array}$ & $\begin{array}{l}\text { Germinated } \\
\text { nuts }(\mathrm{P} 1+\mathrm{P} 2+\mathrm{P} 3)\end{array}$ & Total nuts \\
\hline 17 & 3 & 20 & 27 & & 27 \\
\hline 19 & 1 & 20 & 22 & & 22 \\
\hline 19 & 1 & 20 & 20 & 2 & 22 \\
\hline 20 & - & 20 & 24 & 2 & 26 \\
\hline 20 & - & 20 & 25 & & 25 \\
\hline 21 & - & 21 & 24 & & 24 \\
\hline 20 & 2 & 20 & 24 & & 24 \\
\hline 20 & - & 20 & 23 & 1 & 24 \\
\hline 20 & 3 & 23 & 26 & 2 & 28 \\
\hline 21 & 2 & 23 & 27 & & 27 \\
\hline 20 & 1 & 21 & 19 & 9 & 28 \\
\hline 17 & 3 & 20 & 28 & & 28 \\
\hline 19 & 3 & 22 & 36 & & 36 \\
\hline 20 & 1 & 21 & 29 & 4 & 33 \\
\hline 20 & 2 & 22 & 29 & 1 & 30 \\
\hline 18 & 2 & 20 & 22 & 3 & 25 \\
\hline 18 & 3 & 21 & 32 & 2 & 34 \\
\hline 20 & 1 & 21 & 25 & 1 & 26 \\
\hline 21 & - & 21 & 24 & 1 & 25 \\
\hline 21 & - & 21 & 26 & 5 & 31 \\
\hline 389 & 28 & 417 & 512 & 33 & 544 \\
\hline \multicolumn{3}{|c|}{ Percentage of $i n$-situ germination: $6.71 \%$} & \multicolumn{3}{|c|}{ Percentage of in-situ germination: $6.07 \%$} \\
\hline
\end{tabular}





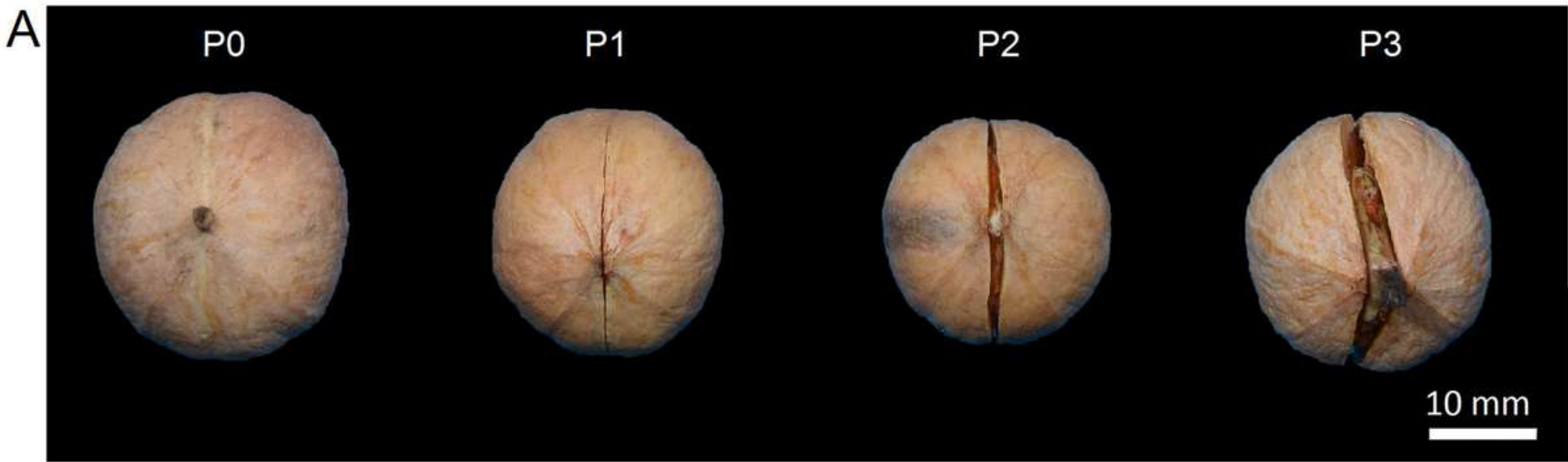

B

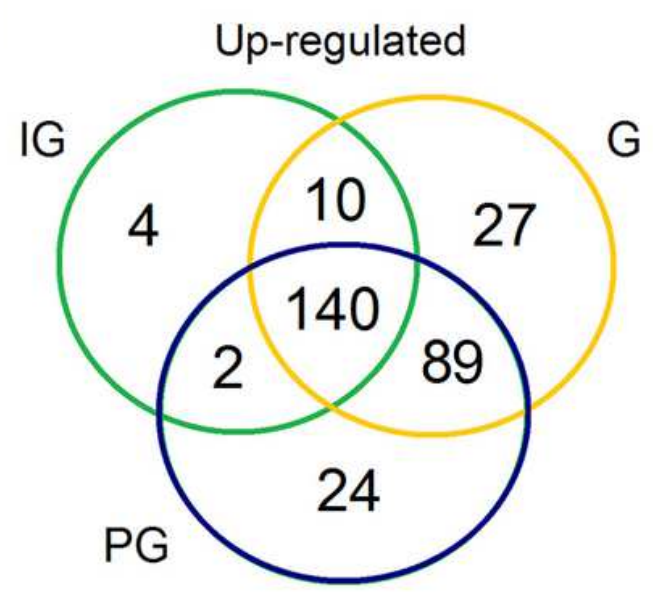

Down-regulated

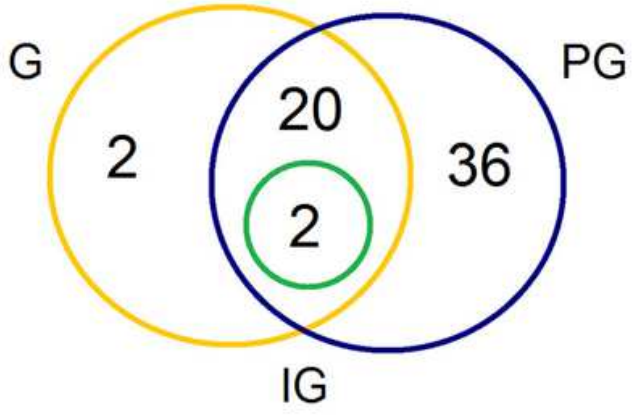

C

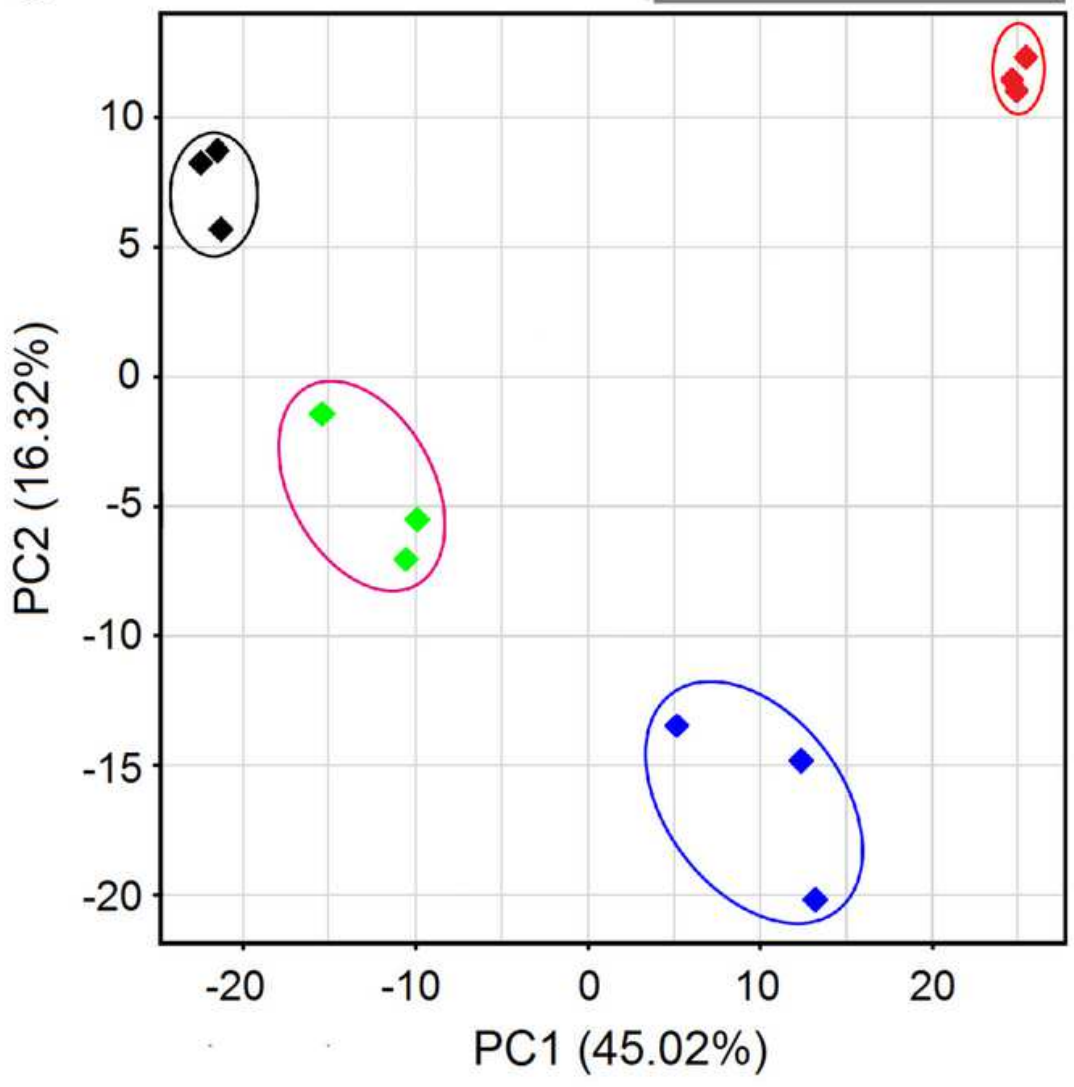

Figure 1

Morphology and overview metabolomic data of Chinese hickory nut during in-situ germination. (A) Phenotype and sample grouping of pre-sprout hickory nuts. Non-germinated nut were assigned as P0. Pre-sprouted nuts with cracked lines on shell were classified as P1, those with white radicle emerged from seed coat were grouped as P2 and pre-sprouts with black radicle tip were grouped as P3. (B) Venn diagram of differentially regulated metabolites in each in-situ germination stages. (C) Principal component analysis (PCA) plot showing the distribution of non-germinated (P0) and germinated Chinese hickory nut samples (P1-P3). 

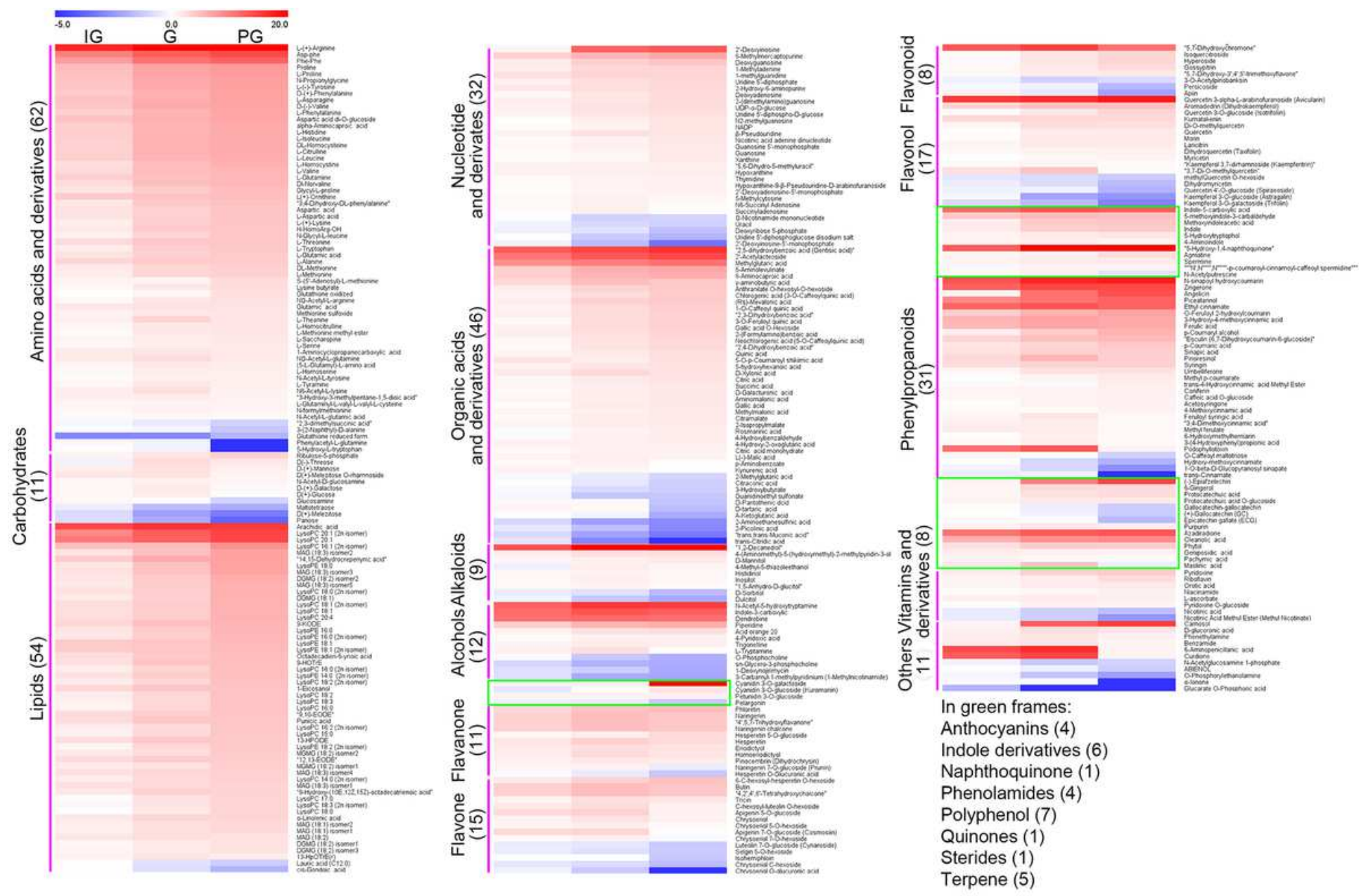

Figure 2

Relative levels and dynamics of differentially regulated metabolites identified during in-situ germination process. The bar indicates the relative levels by log2[FC]. 
A

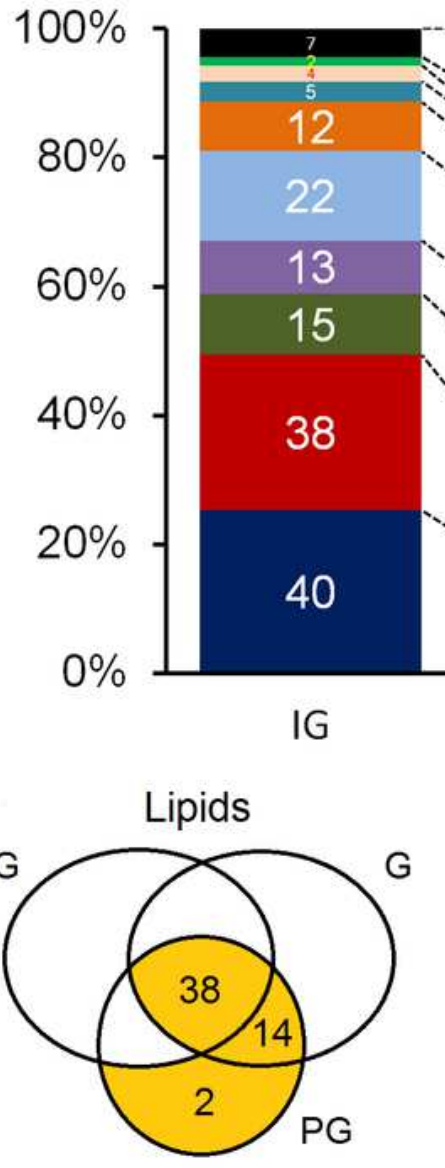

Nucleotide and derivatives
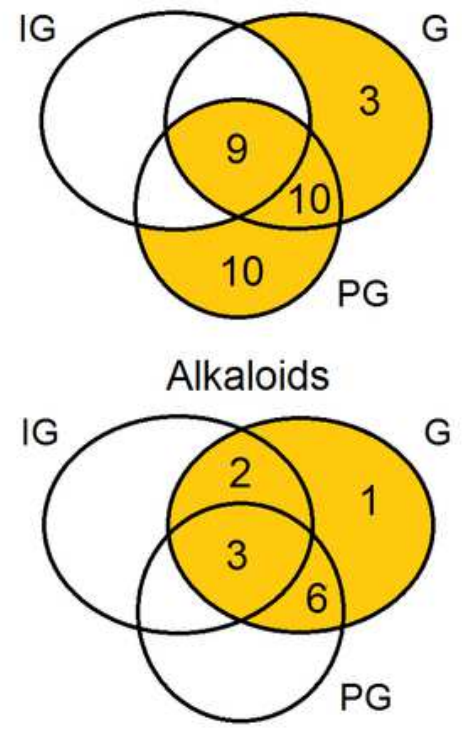
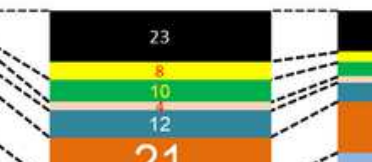

21

41

28

37

52

54

G

Amino acid \& derivatives

IG

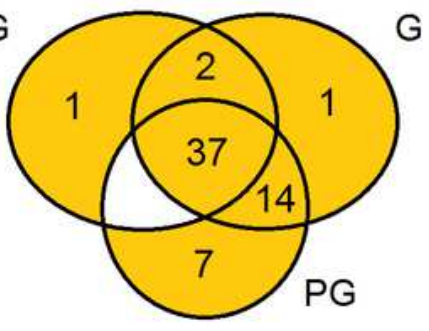

Polyphenols

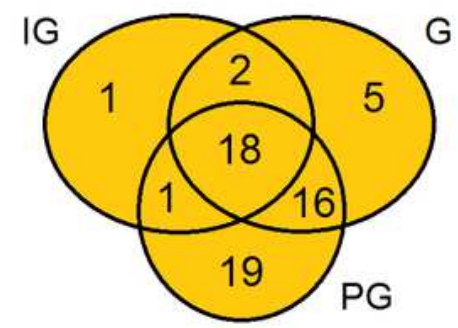

Terpenes

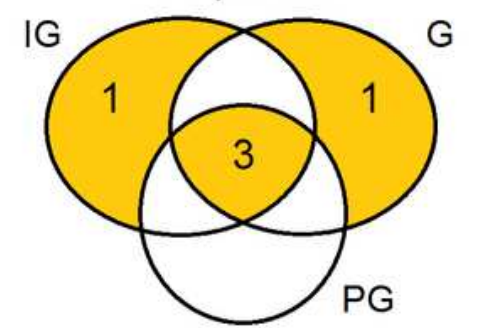

- Others

Alcohols

- Carbohydrates

- Terpenes

- Alkaloids

a Phenylpropanoids

a Polyphenols

n Nucleotides and indoles

- Organic acids and derivatives

- Lipids

- Amino acids and derivatives

Organic acid \& derivatives

IG

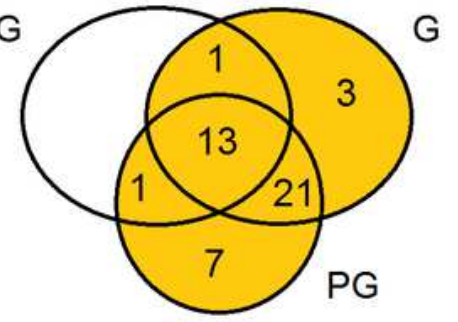

Phenylpropanoids

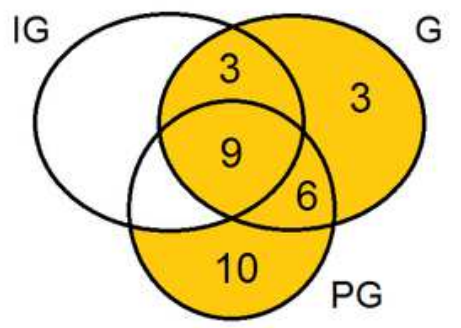

Carbohydrates

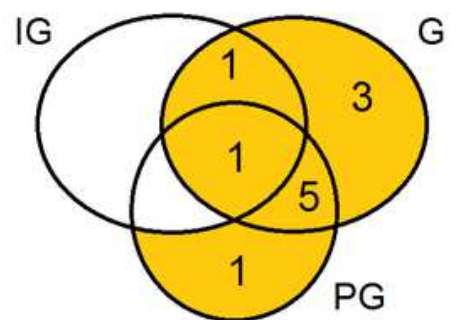

\section{Figure 3}

Classification of differentially-regulated and their distribution in each in-situ germination phases. (A) The Overview of the differentially-regulated classes and their proportions in IG, $G$ and PG phases. (B) Distribution of differentially regulated lipids, amino acids and derivatives, organic acid and derivatives, nucleotides and derivatives, polyphenols and phenylpropanoids during the in-situ germination process. 
A
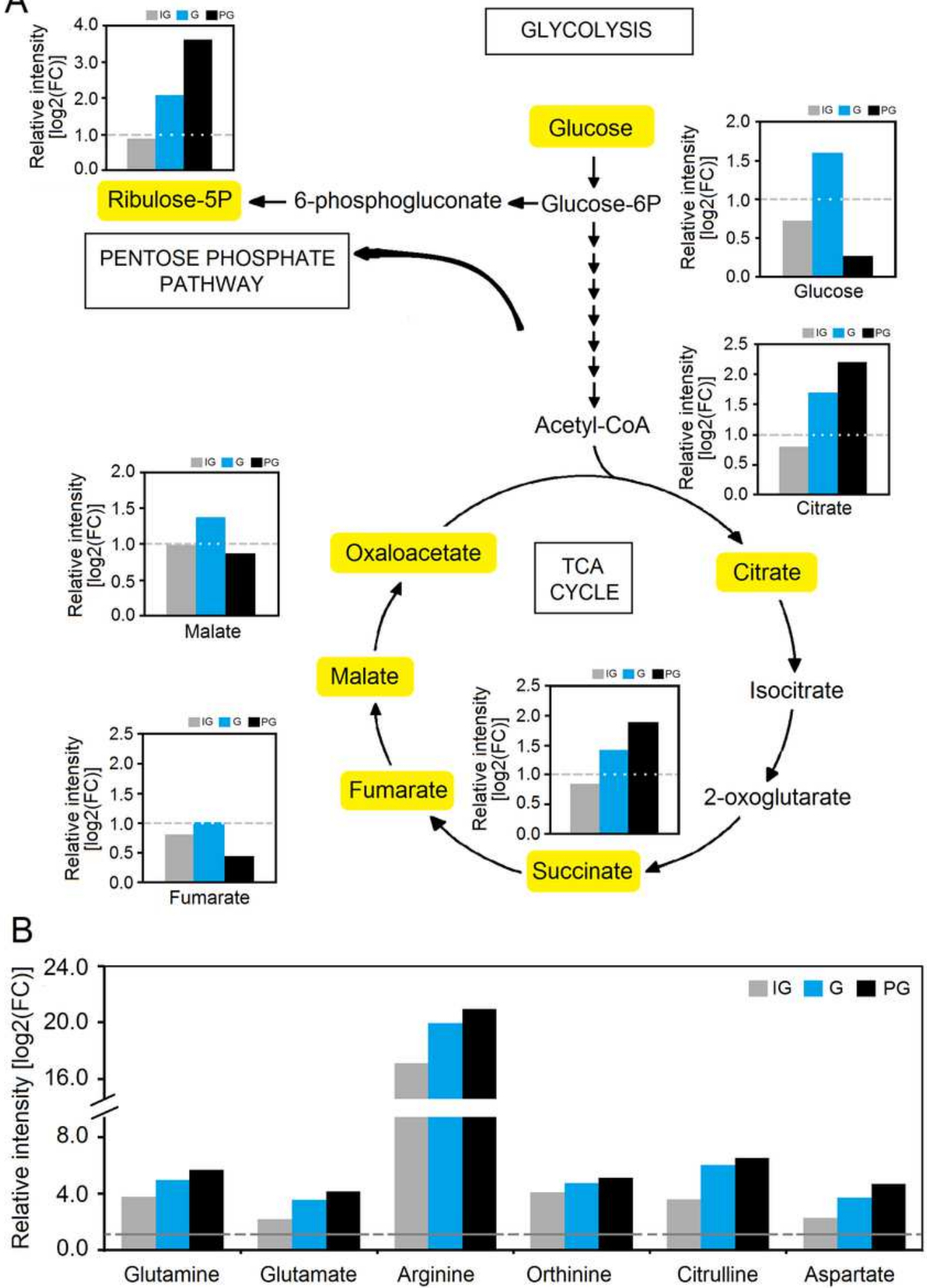

\section{Figure 4}

Dynamic patterns of metabolites involved in managing carbon resource and nitrogen during in-situ germination. (A) The relative abundance of metabolites involved in the glycolysis, pentose phosphate pathway and TCA cycle. (B) The relative abundance of major nitrogen compounds contributors and those involved in urea cycle. 


\section{Supplementary Files}

This is a list of supplementary files associated with this preprint. Click to download.

- TableS1.xlsx

- FigureS1.png 